\title{
Phase Change in Uranium: Discrepancy Between Experiment and Theory
}

Jagan Akella

RECEIVED

AU6 021996

OSTI

July 22, 1996

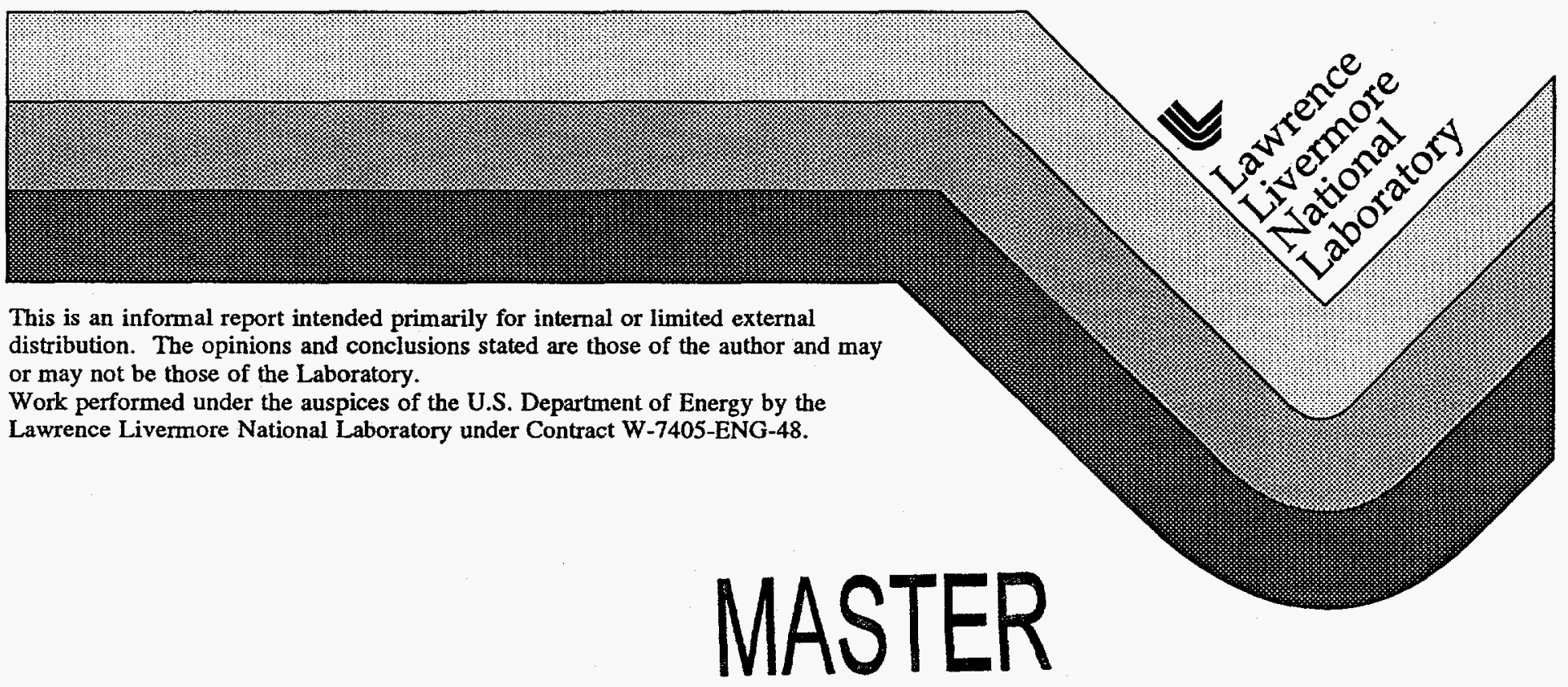




\section{DISCLAIMER}

This document was prepared as an account of work sponsored by an agency of the United States Government. Neither the United States Government nor the University of California nor any of their employees, makes any warranty, express or implied, or assumes any legal liability or responsibility for the accuracy, completeness, or usefulness of any information, apparatus, product, or process disclosed, or represents that its use would not infringe privately owned rights. Reference herein to any specific commercial product, process, or service by trade name, trademark, manufacturer, or otherwise, does not necessarily constitute or imply its endorsement, recommendation, or favoring by . the United States Government or the University of California. The views and opinions of authors expressed herein do not necessarily state or reflect those of the United States Government or the University of California, and shall not be used for advertising or product endorsement purposes.

This report has been reproduced directly from the best available copy.

Available to DOE and DOE contractors from the

Office of Scientific and Technical Information

P.O. Box 62, Oak Ridge, TN 37831

Prices available from (615) 576-8401, FTS 626-8401

Available to the public from the

National Technical Information Service

U.S. Department of Commerce

5285 Port Royal Rd.

Springfield, VA 22161 


\section{DISCLAIMER}

Portions of this document may be illegible in electronic image products. Images are produced from the best available original document. 
Phase Change in Uranium: Discrepancy Between Experiment and Theory Jagannadham Akella, Samuel T. Weir, Gordon S. Smith, and Chantel Ruddle Lawrence Livermore National Laboratory Livermore, CA 94550

\begin{abstract}
Using a diamond-anvil cell (DAC) phase transformation and room temperature Equation of State (EOS) for some actinides and lanthanides were studied to multimegabar (megabar $=100 \mathrm{GPa}$ ) pressures. Experimental data are compared with the theoretically predicted crystal structural changes and the pressure-volume relationships.

There is a general agreement between theory and experiment for the structural changes in the lighter actinides, however in detail there are some discrepancies still. A generalized trend for the phase transformations in the lanthanides can be seen, which again has broad agreement with theory.

We conclude that an accurate and robust theoretical base for predicting the phase transformations in the f-electron metals can be developed by incorporating the DAC data.
\end{abstract}


Introduction: Using a diamond-anvil cell (DAC) phase transformations and room temperature equation of state for uranium have been studied to multimegabar (megabar $=100 \mathrm{GPa}$ ) pressures because it is an important weapons' related material and also to gain a better understanding for the high pressure behavior of $5 f$ elements. ${ }^{1,2,3}$ Söderlind ${ }^{4}$ has recently performed total-energy calculations as a function of volume on uranium. However, both studies have arrived at differing conclusions with regard to structural phase changes in uranium. The purpose of this report is to examine the different conclusions arrived at in these two studies, and discuss what could be done to resolve the issue.

\section{Experimental details}

We used Mao-Bell type diamond-anvil cells. ${ }^{5}$ Diamond-anvils with $300 \mu \mathrm{m}$ culet with $50 \mu \mathrm{m}$ central flat and $7.5^{\circ}$ bevel angle are used. The depleted uranium sample used for these experiments is from an electro-refined rod. X-ray diffraction data were obtained by the energy dispersive method at the National Synchrotron Light Source (NSLS) of the Brookhaven National Laboratory. The resulting accuracy in the calculated volumes from the crystallographic data ranges between 1-2 percent and the sample pressure measured in situ with platinum internal marker are accurate to $\pm 1-1.5 \mathrm{GPa}$. A description of the DAC experimental procedures are detailed elsewhere. 6 


\section{$\underline{\text { Results }}$}

Experimental:

Akella et $\mathrm{al}^{1}$ in their first study of uranium have reported the appearance of two new reflections from an exploratory run made at $71 \mathrm{GPa}$ compared to the ambient spectrum. They surmised further that this could be an indication for a structural phase change in uranium. However, in another study by Akella et $\mathrm{a}^{2}$, to $100 \mathrm{GPa}$, no growth in the intensity of these lines was observed and also no conversion of the low pressure phase totally to a new high pressure phase was seen even at $100 \mathrm{GPa}$. This implied that the appearance of the "two new reflections" could be due to some other reason than a possible structural change.

A most recent study of uranium to multi-megabar pressure (Akella et $\mathrm{al}^{3}$ ) has reconfirmed the above observations to conclude further that indeed there is no high pressure structural change in uranium. Appearance of the "new reflections" is attributed to an anisotropic compression of the crystal axis $a, b$ and $c$. In this context the following observations are relevant.

1. As pressure is increased, compression in the $c$ axial direction is less than in $a$ and $b$.

2. Beyond $70 \mathrm{GPa}$ pressure the relative compressibilities of $a$ and $b$ axis flip and $b$ becomes more compressible than $a$.

3. Initially the (021) and (022) reflections of the ambient orthorhombic uranium phase overlap each other. However, because the (021) line is compressing faster than the (002) line, as the pressure is increased the (002) line emerges from overlap with the (021) line. This accounts for one of the "new reflection," which actually is not due to a phase 
change. The second reflection has a very low intensity and does not show any change.

4. Pressure range at which $a / a o$ and $b / b o$ axial ratios intersect (Fig. 1) is approximately the pressure at which theory predicts bct structure stable.

\section{Theoretical Calculations}

Eriksson and Johansson from Uppsala University, J.M. Wills and colleagues from Los Alamos National Laboratory, and Söderlind and Moriarty from Lawrence Livermore National Laboratory have extensively worked on theoretical calculations to predict the crystal structural changes and pressure volume relationships for actinides.

Wills and Eriksson, 7 and Eriksson, et al. ${ }^{8}$ have done calculations for $\mathrm{Th}, \mathrm{Pa}$, and $\mathrm{U}$, and Th, respectively. Söderlind, et al. ${ }^{9}$ extended these calculations to all the lighter actinides in order to develop a unified picture. These calculations were done by means of first-principles total-energy calculations using the linear 'muffin-tin' orbital method with a self-consistently calculated potential. ${ }^{10}$ The only input data for their calculation, as reported by them, are the atomic number, the crystal structure and the atomic volume.

In the case of thorium, Eriksson, et al..$^{8}$ calculations could confirm the experimentally determined crystallographic phase transition from fcc $\rightarrow$ bct, albeit at a slightly high pressure $100 \mathrm{GPa}$ versus experimental $72 \mathrm{GPa}$. For another lighter actinide $\mathrm{Np}$, the structural sequence with increasing pressure from theory ${ }^{11}$ was orthorhombic $\rightarrow(b c t) \rightarrow$ bcc. Calculations failed to predict a stability region for the bct neptunium phase, however experimental data showed an intermediate phase between the orthorhombic and the bcc, which has a narrow stability region and is 
considered to be a body-centered tetragonal structure (experiments for a positive reconfirmation are planned).

The predicted crystal structure sequence from theory for uranium is alpha $u$ (orthorhombic) $\rightarrow$ bct $\rightarrow$ bcc. The first transformation from alpha uranium to bodycentered tetragonal phase is predicted to be at $80 \mathrm{GPa}$ pressure. As was mentioned already, experimentally in uranium no phase change could be detected even up to multi-megabar pressures at room temperature.

The fortuitous intersection of the $a / a 0$ and $b / b o$ axial ratios at about $100 \mathrm{GPa}$ pressure is approximately the pressure at which bct structure was predicted. However, unfortunately this observation does not confirm the theoretically predicted orthorhombic to bct structural phase change in uranium.

\section{Discussion:}

Even though there is a general agreement between theory and experiment for the structural changes in the lighter actinides, there are discrepancies still in detail. They need to be resolved for a better agreement, so that theory can predict materials' properties to tens of megabar pressures.

The discrepancy between theoretical prediction and the experimental observation regarding the structural change in uranium is noteworthy. Söderlind, et al ${ }^{4}$ used fixed values of $b / a$ and $c / a$ (as observed at ambient pressure) in their total energy calculations on the orthorhombic phase. Presumably, optimized $b / a$ and $c / a$ values would lower the orthorhombic total energy at high pressure, possibly eliminating the intermediate bct phase. It is also interesting to note that so far uranium is the only lighter actinide which does not go through a structural phase change at room temperature and high pressures. 
If the structural change is an extremely sluggish phase transformation at room temperature, possibly it could be missed experimentally. In such a case, if experiments are conducted at slightly elevated temperatures (e.g. ohmic heating), the transformation may be accelerated. A concurrent effort to do theoretical calculations utilizing the experimentally determined axial and volume compression data (Fig. 2) under pressure could resolve the structural transformation discrepancy, and may also shed light on the differences in the high pressure behavior of uranium compared to other lighter actinides.

\section{Acknowledgments}

The authors would like to thank Drs. John Moriarty, Richard Grover, John Wills, Olle Eriksson, Per Söderlind, and Börje Johansson for critical discussions. Funding for this work is provided by the B-Division at Lawrence Livermore National Laboratory under DAC actinides research project. We are grateful to Drs. Bruce Goodwin and Mike Anastasio for their constant encouragement and financial support. Work performed under the auspices of the U.S. Department of Energy by Lawrence Livermore National Laboratory under Contract W-7405-ENG-48.

\section{References:}

1. J. Akella, G.S. Smith, and H. Weed, J. Phys. Chem. Solids, 46, 399 (1985).

2. J. Akella, G.S. Smith, R. Grover, Y. Wu, and S. Martin, High Pressure Research, 2, 295 (1990).

3. J. Akella, S. Weir, and G. Smith, Multi Megabar Static Equation-of-State of Uranium using a Diamond-Anvil Cell (DAC), UCRL-ID (1993).

4. P. Söderlind, Theory for the crystal structures of cerium and the light actinides at low temperature and high pressure (draft)

5. H.K. Mao, and P.M. Bell, Carnegie Institution of Washington, Geophys. Lab. Yearbook 74402 (1975). 
6. J. Akella, S.T. Weir, and B. Goodwin, Science \& Tech. Review (LLNL), March 1966.

7. J.M. Wills, and O. Eriksson, Phys. Rev. B. 4513879 (1992).

8. O. Eriksson, J.M. Wills, and P. Söderlind, fcc-bct phase transition in Th at extreme compressions: Theory, Los Alamos UR-92-289 (1992).

9. P. Söderlind, O. Eriksson, B. Johansson, J.M. Wills, and A.M. Boring, Nature 374 (1995).

10. J.M. Wills, and B.R. Cooper, Phys. Reo. B 362809 (1987).

11. P. Söderlind, B. Johansson, and O. Eriksson, Phys. Rev. B 52, 1631 (1995).

\section{Figure Captions:}

Fig. 1 The axial ratios for uranium plotted as a function of pressure. Note the change in the relative compressibilities of $a$ and $b$ axis.

Fig. 2 Volume compression data $\mathrm{V} / \mathrm{V}_{\mathrm{o}}$ plotted against pressure. 

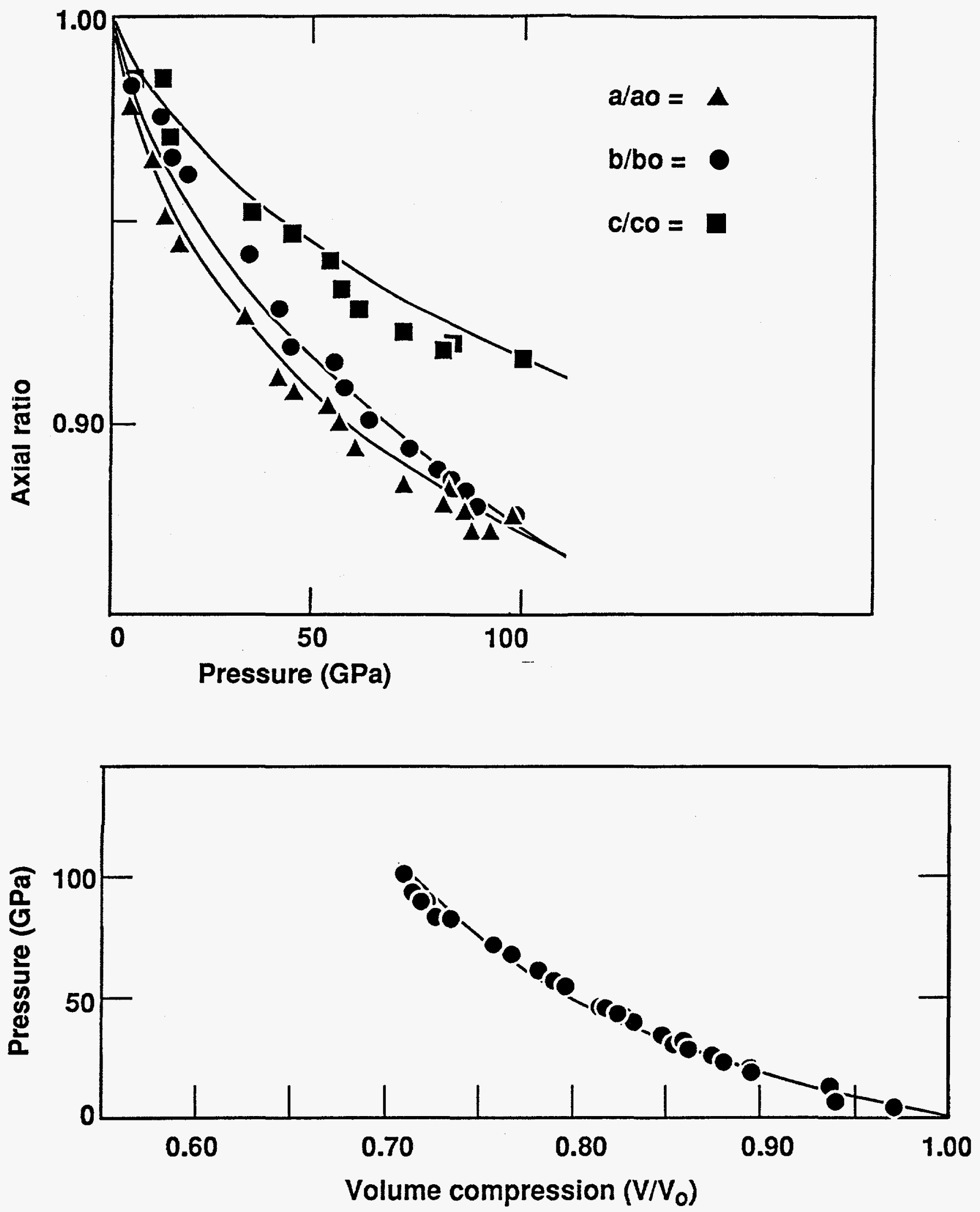


\section{Distribution:}

Mike Anastasio, L-160

James Belak, L-50

Richard Fortner, L-51

Jay C. Davis, L-103

Thomas J. Gilmartin, L-001

Lewis Glenn, L-200

Bruce Goodwin, L-170

Richard Grover, L-50

W. J. Hannon, L-203

Rob Hickson, LANL

Leon Keller, L-170

David Lassila, L-170

Ronald Lee, L-281

Christian Mailhiot, L-45

Andy McMahan, L-45

John A. Moriarty, L-50

Chantel Ruddle, L-364

Bruce Tarter, L-001

Harry Vantine, L-170

Thomas Weaver, L-45

Larry Wiley, L-170

Samuel Weir, L-281

John M. Wills, LANL

David A. Young, L-50 

Technical Information Department - Lawrence Livermore National Laboratory

University of California • Livermore, California 94551

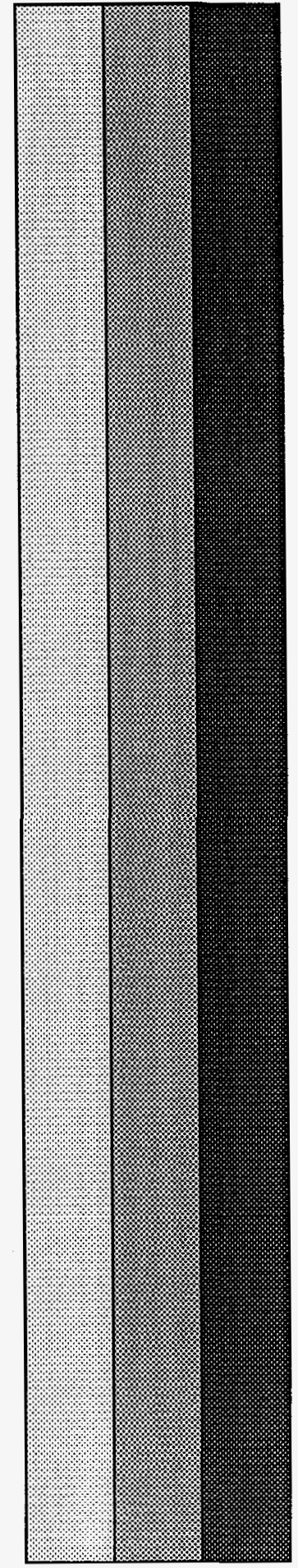

